\title{
Jobs-Housing Imbalance, Spatial Correlation, and Excess Commuting
}

\author{
by
}

Tsutomu SUZUKI ${ }^{\mathrm{a}^{*}}$ and Sohee $\mathrm{LEE}^{\mathrm{b}}$

${ }^{a^{*}}$ Corresponding author. Department of Risk Engineering, Graduate School of Systems and Information Engineering, University of Tsukuba

1-1-1 Tennodai, Tsukuba, Ibaraki 305-8573, Japan

E-mail: tsutomu@risk.tsukuba.ac.jp

Tel. \& Fax: +81-29-853-5186

${ }^{\mathrm{b}}$ Center for Spatial Information Science, University of Tokyo

5-1-5 Kashiwanoha, Kashiwa, Chiba 277-8568, Japan

E-mail: shlee@csis.u-tokyo.ac.jp

\section{Acknowledgement}

This work was partially funded by Grants in Aid for Scientific Research awarded by the Japan Society for the Promotion of Science (JSPS) and by the Asahi Glass Foundation research grant program. 


\begin{abstract}
In this paper, we use continuous urban structure instead of zonal model, try to calculate unbiased excess commuting with joint distribution of homes and workplaces developed by Vaughan (1974), and describe the relationship between urban structure and commuting distance explicitly and theoretically for generalized home-workplace assignment pattern. We simplify the quadrivariate distribution model to a model with three important parameters: the spread of homes, the spread of workplaces, and the spatial correlation of homes and workplaces. Then we show that excess commuting and capacity utilization are expressed by the imbalance and the spatial correlation of jobs-housing structure in a theoretical context, moreover it explicitly evaluates targeting US and Japanese/Korean cities.
\end{abstract}

Keywords: jobs-housing balance, spatial correlation, commuting efficiency, excess commuting, urban structure 


\section{Introduction}

Many researchers have challenged the measurement of excess commuting, which is calculated as the difference between the actual and minimum average commuting distances (or times). The latter is obtained by solving the transportation problem using linear programming, with the distribution of homes and workplaces being fixed. Excess commuting may be interpreted as the commuting that can be eliminated by adjusting the locations of homes or workplaces. If the excess is large, the commuting distance (or time) can be drastically reduced by controlled matching of homes and workplaces.

Since White (1988) re-examined the fundamental assumption of cost minimization by applying linear programming, many empirical studies have been carried out in this regard. These studies aim to estimate the excess commuting in sample cities and assess the usefulness of urban policies intended to balance jobs and housing (e.g., Frost et al., 1998; Kim, 1995; Merriman et al., 1995; O'Kelly and Lee, 2005; Small and Song, 1992).

Other studies on excess commuting have provided methodological extensions, especially on the conceptual side. In particular, researchers have introduced the concept of maximum commute, which is calculated using linear programming, to maximize the commuting cost for trips between homes and workplaces (e.g., Boussauw et al., 2011; Charron, 2007; Horner, 2002; Layman and Horner, 2010; Ma and Banister, 2006b; Murphy, 2009; Murphy and Killen, 2010). The theoretical range of the commute, i.e., the difference between the maximum and minimum commutes, is the available commuting potential of a city. The ratio of the actual excess commute and the theoretical commute range is an indicator of commuting efficiency.

However, in the aforementioned studies, the researchers could not decide how the urban structure, i.e., the distribution of homes and workplaces, influences excess commuting. Merriman et al. (1995) carried out a simulation study to show that any structural change toward the decentralization of employment centers would result in an increase in excess commuting. Ma and Banister (2007) discussed the relationship between changes in the trip length and urban form, as well as that between changes in the urban form and urban commuting capacity, in relation to the measurement of urban spatial change and excess commuting. However, as pointed out by Horner and Murray (2002), in the abovementioned researches, the modifiable areal unit problem was overlooked. This was because the exact extent of the excess was not determined, which in turn was because of the imperfect measurement methodology used: a zonal approach that yields aggregation biases.

The estimated value of excess commuting is very sensitive to errors in the measurement method, geographical boundaries, and data resolution, as well as to differences in the individual commuting behavior across various cities. For this reason, it is difficult to draw general conclusions from empirical studies when this approach is used.

Very few theoretical studies have been carried out to evaluate the best employee distribution for a desirable urban structure. One reason for this is that it is difficult to express clearly the spatial distribution of homes and workplaces and its relation to commuting. Therefore, in this study, we adopt an analysis method based on Vaughan's (1974) joint distribution of homes and workplaces. Although this model can be used to express such complicated relations at a macro level, it has not been used frequently thus far.

This study aims to describe the relationship between urban structure and commuting distance explicitly and theoretically for generalized home and workplace assignment patterns. Accordingly, we try to calculate the unbiased excess commuting by using a joint distribution model of homes and workplaces and a continuous urban structure instead of a zonal model.

Section 2 presents a review of the debate on excess commuting. In Section 3, we describe a model to verify the relation between urban structure and commuting distance. First, we simplify the quadrivariate distribution model (Vaughan, 1974) to a model with three important parameters: (1) spread of homes, (2) spread of workplaces, and (3) the spatial correlation between homes and workplaces. Second, we show that the average commuting 
distance can be evaluated explicitly by the abovementioned three parameters and that excess commuting and capacity utilization can be theoretically expressed by the imbalance and spatial correlation between the jobs and housing structure. In Section 4, we present our findings along with a comparison of the results obtained for some US and Japanese/Korean cities. Section 5 presents our conclusions.

\section{Debate on excess commuting and its extensions}

Excess commuting is defined as the difference between the actual and the minimum average commute for a given distribution of homes and workplaces. By measuring excess commuting, we can determine the extent to which commuting distance or time is an inevitable result of the functioning of a vast interconnected economic system and the extent to which it is the result of inefficient matching of homes and workplaces.

Hamilton (1982) carried out a test on excess commuting using a monocentric model in which the workers were assumed to be distributed according to a Clark-type model. He concluded that for several US cities, the excess was $80 \%$ or more of the actual commuting distance. He focused on the fact that commuters do not necessarily minimize their commuting costs, as is often assumed in models of urban economics.

White (1988) adopted a zonal approach. She divided the targeted area into several zones and calculated the flow using an origin-destination matrix. She derived the minimum average commuting time by solving a transportation problem. After carrying out tests on some US cities, she evaluated the excess to be about $10 \%$ criticizing the inability of Hamilton's monocentric model to account for the actual distribution of residences and workplaces, but her method included an aggregation bias. Hamilton (1989) and Small and Song (1992) corrected the bias and found the excess to be more than $60 \%$.

Following Hamilton (1982, 1989) and White's (1988) study on the concept and estimation of excess commuting, many empirical studies have been carried out for different cities. For example, excess commuting in the Tokyo metropolitan area was studied by Merriman et al. (1995). In the zonal approach, the urbanized area within $60 \mathrm{~km}$ of the center of Tokyo was divided into 211 jurisdictional zones and the minimum average commuting time was $42 \mathrm{~min}$, whereas the average observed commuting time was 49 min. Thus, the excess was $15 \%$, which was significantly less than the actual figure for US cities. The excess increased to $36 \%$ when commuting distance was used instead of commuting time. In US cities, however, the excess was found to be above $60 \%$ even when commuting distance was used.

Frost et al. (1998) studied excess commuting in a selection of UK cities. Kim (1995) developed models that predict the commuting distances for two-worker households and estimated the excess commuting in Los Angeles. O'Kelly and Lee (2005) developed a trip distribution model that disaggregates journey-to-work data according to occupation type, in order to estimate actual commutes and to measure the theoretical minimum and maximum commutes via a linear program. They reported variations in the excess commuting and the jobs-housing balance for different occupation types.

Recently, Horner (2010) also focused various worker groups and looked at the matter that in general the theoretical minimum commute might be overestimated using aggregated data within the measurement of excess commuting. To clarify the error potential in the minimum commute calculation by using disaggregating worker data, several computational calculations were estimated based all possible combinations on the proportional allocation method and random allocation method. Much larger theoretical minimum commutes gained by disaggregating worker data into finer groupings.

Several trials extending or reinterpreting the excess commuting method have been carried out using the transportation optimization problem. The first trial was performed by Black and Katakos (1987). They considered the urban spatial structure for which the commute 
is maximized by using an optimization method; this method is equivalent to that proposed in previous studies on excess commuting. To classify different urban structures, they calculated an urban consolidation index, which is defined as the ratio of the minimum and maximum commuting distances for a given city.

Horner (2002) introduced the theoretical maximization concept to provide insights into the degree of decentralization of an urban form. He suggested an index commuting potential consumed, which is identical to capacity utilization mentioned in this paper and is an indication of how much of the available commuting range has been consumed. Commuting potential consumed is defined as the ratio of the difference between the observed and theoretical minimum commuting distances to that between the theoretical maximum and minimum commuting distances. This index is useful for comparing the commuting efficiency of cities because it is an improvement over the traditional definition of excess commuting. Horner (2002) also showed the variation in excess commuting for a selection of US cities and the relative use of commuting resources in each city. He showed that the commuting potential of these cities did not reach the upper limit, although the excess values for the cities were drastically different.

Following Horner's approach, Murphy (2009) analyzed the excess commuting in Dublin for 1991 and 2001 and investigated the potential impact of increases in the network density for public and private transport. He showed that public-transport users can dramatically reduce their commuting distance if the density of the public-transport network is increased considerably.

Charron (2007) proposed a new framework for excess commuting, introducing the concept of random average commute and the distribution of commuting possibilities. The random average commute, the value of which lies usually between the observed and maximum commuting distances, is the most probable commuting outcome (theoretically) if commuting behavior is not influenced by distance. In this framework, the observed commuting distance is lower than the average value of the commuting possibilities (the random average commute). Fifty metropolitan areas of the US were studied to find the dynamic relationship between the spatial behavior and the urban form. Most recently, Murphy and Killen (2011) also applied the random commuting concept for measuring the efficiency of regional commuting patterns. They considered the average random commute is a more appropriate base measure because it relates solely to a specific type of behavior where cost is irrelevant in decision-making. They proposed two new measures of commuting efficiency: commuting economy and normalized commuting economy. On this basis, they found out that the average actual commute has moved further away from the average random commute, implying that greater intermixing of jobs and housing has led to more efficient commuting behavior in the Greater Dublin Area.

There has been a debate on the methodological issues concerning the measurement of excess commuting using linear programming (Horner, 2004; Ma and Banister, 2006a). For example, Horner and Murray (2002) claimed that aggregation and the spatial unit definition (the modifiable areal unit problem) affected the estimation of excess commuting. In many applications, the commuting cost is considered together with the commuting time or distance (Euclidean or network distance), and this directly affects the estimation. The estimation is also sensitive to the spatial definition of the zonal units. In this regard, Horner (2010) discussed the grey transportation costs, which are defined as the lower and upper bounds on the travel time between a given origin and destination.

Some researchers tried to clarify the relationship between excess commuting and spatial distribution patterns (spatial structure). Ma and Banister (2006b) suggested an extended excess commuting technique involving the calculation of the maximum and minimum commutes, which indicate the quantitative and qualitative imbalance between workplaces and residential locations with respect to the urban spatial dispersal. Quantitative imbalance is defined as the difference between the minimum and zero commutes. Thus, quantitative balance (or numerical equality) signifies that the physical land use is well mixed. Qualitative imbalance 
is defined as the difference between the observed and minimum commutes; this difference is conventionally referred to as excess commuting. Ma and Banister (2007) adopted the framework of Brotchie's urban triangle model (Brotchie, 1984; Brotchie et al., 1996), which provides useful insights into the relationship between urban spatial change and urban travel. They also explained the extended excess commuting measure, proposed the use of both minimum and maximum levels to understand the concept of commuting potential, and discussed the relationship between commuting potential and urban form. Furthermore, Boussauw et al. (2011) detected the process of spatial separation using a time series approach of the minimum commuting distance that measured in the excess commuting framework. Niedzieski (2006) introduced spatially disaggregated measures of commuting efficiency including excess commuting that allows us to consider zonal commuting efficiency in greater spatial detail.

Most recently, Layman and Horner (2010) attempted to investigate connections between jobs-housing balance that is expressed as the metrics of worker and job locations, and transportation. It also has developed from the concept of excess commuting to seek to the urban structural changes. In their paper, the theoretical minimum and maximum commutes, and the random average commute on the job and worker growth scenarios are computed to explore the relationships between these interrelated metrics.

Three issues have been identified on the basis of this literature review. The first is the definition of the commuting cost. Usually, distance or time is used to determine excess commuting, and the result varies depending on the parameter chosen. The excess calculated using the commuting distance is larger than that calculated using commuting time (Merriman et al., 1995). Moreover, the commuting distance can be measured using the Euclidean distance or the actual network distance. For example, Giuliano and Small (1993), Ma and Banister (2006b), and Murphy (2009) used the network distance for obtaining more reliable results.

Second, excess commuting is caused by multiple factors, including jobs-housing imbalance. Direct comparison of the excess commuting values of different cities is difficult because of variations in the deciding factors across the cities, such as household characteristics, job locations, occupation types, housing prices, and housing locations.

Third, the modifiable areal unit problem (MAUP) should not be overlooked. As aggregation occurs or the number of zones decreases, the range of the excess commuting estimates becomes smaller (Horner and Murray, 2002). Using a small number of zones gives poor results because the excess commuting calculated by solving the transportation problem is small. Thus, disaggregate and deductive approaches should be considered. The traditional zonal approach is based on a network representation associated with a discrete set of coordinates such as locations of jurisdictional centers. Every point in geographic space is assigned to a particular zone, and every zone is associated with one or more nodes in the network. This approach often causes aggregation problems such as biases in the measurement of excess commuting.

In this study, we will focus especially on the third issue. We show theoretically that the commuting distance can increase or decrease depending on the jobs-housing balance and the correlation between the locations of homes and workplaces. If the urban space is represented as a field, we no longer need to consider a zonal system or a transportation network. Distributions of the population, employment, and spatial interactions such as commuting trips are given as continuous distributions. This approach can be used for a simple and economical estimation of the spatial pattern of travel in a city and is particularly suitable for macroscopic studies. This approach is a complement to the traditional transportation planning methods rather than an alternative.

Continuous distribution is never a new approach. Clark distribution that Hamilton (1982) used in his work is the most typical continuous function of population or employment. However, in this paper, we introduce not only spatial distribution of workers but also spatial correlation between homes and workplaces - that means commuting distance is not decided only by spatial structure. In this paper, we will show that it is possible to draw a more realistic commuting patterns including reverse commuting by introducing one more dimension - 
correlation between homes and workplaces, even though we use a theoretical modeling approach.

\section{Description of the relation between urban structure and commuting distance}

\subsection{Joint distribution of homes and workplaces}

In this section, we define home as the home end of a work trip and workplace as the destination end. The total number of homes and workplaces is denoted by $P$.

Vaughan (1987) summarized the notion of the joint distribution of homes and workplaces that he formulated in 1974. Usually, administrative zones are used to describe the movement of commuters from one part of a city to another. However, in the continuous approach adopted here, we use mathematical expressions to find the number of trips between any two unit zones.

Continuous urban models have been extensively researched. Clark (1951) approximated residential densities by a negative exponential function, and Alonso (1964) suggested that rents decrease with an increase in the distance from the city center. The distribution of population or rents and the spatial interaction of the geometrical representation are associated with a continuous coordinate system. Angel and Hyman (1972) established a radially symmetric continuous model to represent the spatial distribution of accessibility to jobs and homes and that of traffic flow. They introduced the notion of the trip density function, the number of trips from a unit area at an origin to a unit area at a destination; this notion is similar to our concept of the joint distribution of homes and workplaces.

Haight (1964) carried out research on the trip length between two points in a continuous context. Fairthorne (1965) considered the average distance between pairs of points and used it to measure the accessibility of homes and workplaces with various routing systems, including direct routing.

The analysis in this study is based on Vaughan's (1974) joint distribution of homes and workplaces. Wilkins (1969) took the first step to the development of a realistic model by assuming that homes and workplaces were distributed according to Sherratt-type model, which is identical to the two-dimensional normal distribution, but uncorrelated. Vaughan (1974) realized that a spatial correlation effect could be introduced when using the quadrivariate normal distribution that allows homes and workplaces to remain individually distributed in accordance with Sherratt-type model. This continuous model of work trips can be used in urban-commuting models.

Vaughan's (1974) joint distribution of homes and workplaces can be written as a general quadrivariate normal distribution:

$$
\left.f_{h w}\left(x_{h}, x_{w}, y_{h}, y_{w}\right)=\frac{P}{(2 \pi)^{2} \sqrt{|\mathbf{V}|}} \exp \left[-\frac{1}{2}(z-\boldsymbol{\mu})^{\mathrm{T}} \mathbf{V}^{-1}(z-\boldsymbol{\mu})\right)\right],
$$

where $\left(x_{h}, y_{h}\right)$ and $\left(x_{w}, y_{w}\right)$ represent the locations of homes and workplaces in Cartesian coordinates; $P$, the total number of employees; $z^{\mathrm{T}}=\left(x_{h}, x_{w}, y_{h}, y_{w}\right)$, the location vector; and $\boldsymbol{\mu}^{\mathrm{T}}=\left(\mu_{x_{h}}, \mu_{x_{w}}, \mu_{y_{h}}, \mu_{y_{w}}\right)$, the location vector of the centroids.

$$
\mathbf{V}=\left(\begin{array}{cccc}
\sigma_{x_{h}}^{2} & \operatorname{Cov}\left(x_{h}, x_{w}\right) & \operatorname{Cov}\left(x_{h}, y_{h}\right) & \operatorname{Cov}\left(x_{h}, y_{w}\right) \\
\operatorname{Cov}\left(x_{h}, x_{w}\right) & \sigma_{x_{w}}^{2} & \operatorname{Cov}\left(x_{w}, y_{h}\right) & \operatorname{Cov}\left(x_{w}, y_{w}\right) \\
\operatorname{Cov}\left(x_{h}, y_{h}\right) & \operatorname{Cov}\left(x_{w}, y_{h}\right) & \sigma_{y_{h}}^{2} & \operatorname{Cov}\left(y_{h}, y_{w}\right) \\
\operatorname{Cov}\left(x_{h}, y_{w}\right) & \operatorname{Cov}\left(x_{w}, y_{w}\right) & \operatorname{Cov}\left(y_{h}, y_{w}\right) & \sigma_{y_{w}}^{2}
\end{array}\right)
$$

is the variance-covariance matrix of the coordinates, in which the standard deviation of $x_{h}$ is 
denoted by $\sigma_{x_{h}}^{2}$ and the covariance of two coordinates $x_{h}$ and $y_{w}$ is denoted by $\operatorname{Cov}\left(x_{h}, y_{w}\right)$. $\sigma_{x_{h}}^{2}$ indicates the spread of homes in the $x$ direction and can be derived by

$$
\begin{aligned}
\sigma_{x_{h}}^{2} & =E\left[\left(x_{h}-\mu_{x_{h}}\right)^{2}\right] \\
& =\int_{-\infty}^{\infty} \int_{-\infty}^{\infty} \int_{-\infty}^{\infty} \int_{-\infty}^{\infty}\left(x_{h}-\mu_{x_{h}}\right)^{2} f_{h w}\left(x_{h}, x_{w}, y_{h}, y_{w}\right) \mathrm{d} x_{h} \mathrm{~d} x_{w} \mathrm{~d} y_{h} \mathrm{~d} y_{w} .
\end{aligned}
$$

The variances $\sigma_{x_{w}}^{2}, \sigma_{y_{h}}^{2}$, and $\sigma_{y_{w}}^{2}$ are defined similarly. The covariance of two variables, for instance $x_{h}$, and $y_{w}$, is given by

$$
\begin{aligned}
\operatorname{Cov}\left(x_{h}, y_{w}\right) & =E\left[\left(x_{h}-\mu_{x_{h}}\right)\left(y_{w}-\mu_{y_{w}}\right)\right] \\
& =\int_{-\infty}^{\infty} \int_{-\infty}^{\infty} \int_{-\infty}^{\infty} \int_{-\infty}^{\infty}\left(x_{h}-\mu_{x_{h}}\right)\left(y_{w}-\mu_{y_{w}}\right) f_{h w}\left(x_{h}, x_{w}, y_{h}, y_{w}\right) \mathrm{d} x_{h} \mathrm{~d} x_{w} \mathrm{~d} y_{h} \mathrm{~d} y_{w} .
\end{aligned}
$$

with similar definitions for the other pairs of coordinates. The marginal densities of homes and workplaces derived from (1) become Sherratt's normal distributions (nonuniform, infinite boundary, radially symmetric, exponentially decreasing functions), which are convenient to handle mathematically. Blumenfeld and Weiss (1974) and Blumenfeld (1977) showed that Sherratt-type model was more suitable for representing the distribution of homes in UK cities. They also found a good fit for the distribution of workplaces.

\subsection{Simplification}

The above quadrivariate normal joint distribution model was simplified by Vaughan (1974). The model contains fourteen parameters, but under reasonable assumptions, the number of parameters reduces to three. The first simplification is centralization. The centroid of homes and can be made to coincide with the centroid of workplaces by setting

$$
\boldsymbol{\mu}=\mathbf{0}
$$

without loss of generality.

Second, we can simplify the joint distribution model by assuming identical direction of growth. If the directions of growth of homes and workplaces are the same, the covariance of the $x$ and $y$ coordinates of a home and a workplace can be set to zero. Therefore,

$$
\operatorname{Cov}\left(x_{h}, y_{h}\right)=\operatorname{Cov}\left(x_{w}, y_{w}\right)=0 .
$$

Similarly, if every point is equally accessible from every other point in the city, it is likely that the covariance between the $\mathrm{x}$ coordinate of a home and the $\mathrm{y}$ coordinate of a workplace and that between the $\mathrm{x}$ coordinate of a workplace and the $\mathrm{y}$ coordinate of a home will be zero. Therefore,

$$
\operatorname{Cov}\left(x_{h}, y_{w}\right)=\operatorname{Cov}\left(x_{w}, y_{h}\right)=0 \text {. }
$$

The most important covariances are those between the home and workplace positions of an individual in two directions, given by $\operatorname{Cov}\left(x_{h}, x_{w}\right)$ and $\operatorname{Cov}\left(y_{h}, y_{w}\right)$. These variables indicate a worker's desire to live close to his/her workplace in the $x$ and $y$ directions, respectively. We transform them to scale-free measures, i.e., the correlations are given by

$$
\rho_{x}=\frac{\operatorname{Cov}\left(x_{h}, x_{w}\right)}{\sigma_{x_{h}} \sigma_{x_{w}}},
$$

and

$$
\rho_{y}=\frac{\operatorname{Cov}\left(y_{h}, y_{w}\right)}{\sigma_{y_{h}} \sigma_{y_{w}}} .
$$

These variables satisfy

$$
\left|\rho_{x}\right|<1,\left|\rho_{y}\right|<1 .
$$

Moreover, if we assume circularly symmetric correlation, i.e., no bias of development toward the $x$ or $y$ directions, the spreads of homes and workplaces in the two directions take a common value. 


$$
\begin{aligned}
& \sigma_{x_{h}}^{2}=\sigma_{y_{h}}^{2}=\sigma_{h}^{2} \\
& \sigma_{x_{w}}^{2}=\sigma_{y_{w}}^{2}=\sigma_{w}^{2}
\end{aligned}
$$

Similarly, by assuming symmetric correlation, we have

$$
\rho_{x}=\rho_{y}=\rho
$$

so that the variance-covariance matrix can be rewritten as

$$
\mathbf{V}=\left(\begin{array}{cccc}
\sigma_{h}^{2} & \rho \sigma_{h} \sigma_{w} & 0 & 0 \\
\rho \sigma_{h} \sigma_{w} & \sigma_{w}^{2} & 0 & 0 \\
0 & 0 & \sigma_{h}^{2} & \rho \sigma_{h} \sigma_{w} \\
0 & 0 & \rho \sigma_{h} \sigma_{w} & \sigma_{w}^{2}
\end{array}\right),
$$

which has only three parameters. This is the third simplification. Hence, the joint distribution of homes and workplaces can be rewritten as

$$
\begin{aligned}
& f_{h w}\left(x_{h}, x_{w}, y_{h}, y_{w}\right) \\
& =\frac{P}{(2 \pi)^{2} \sigma_{h}^{2} \sigma_{w}^{2}\left(1-\rho^{2}\right)} \exp \left[-\frac{1}{2\left(1-\rho^{2}\right)}\left(\frac{x_{h}^{2}+y_{h}^{2}}{\sigma_{h}^{2}}+\frac{x_{w}^{2}+y_{w}^{2}}{\sigma_{w}^{2}}+\frac{2 \rho\left(x_{h} x_{w}+y_{h} y_{w}\right)}{\sigma_{h} \sigma_{w}}\right)\right] .
\end{aligned}
$$

This model is the simplest among those developed to date and takes into account the three basic factors affecting the distribution of commuting trips: the spread of homes $\sigma_{h}$, the spread of workplaces $\sigma_{w}$, and the spatial correlation $\rho$ between home and workplace locations. The studies by Vaughan (1974) and Blumenfeld (1977) indicate that the simplification is reasonable if the city does not have a geographical peculiarity. Blumenfeld (1977) and other researchers attempted to obtain the maximum likelihood estimates of the three parameters.

The marginal densities of homes and workplaces derived from (15) are given by the following Sherratt-type models:

$$
\begin{aligned}
& f_{h}\left(x_{h}, y_{h}\right)=\int_{-\infty}^{\infty} \int_{-\infty}^{\infty} f_{h w}\left(x_{h}, x_{w}, y_{h}, y_{w}\right) \mathrm{d} x_{w} \mathrm{~d} y_{w}=\frac{P}{2 \pi \sigma_{h}^{2}} \exp \left(-\frac{1}{2} \frac{x_{h}^{2}+y_{h}^{2}}{\sigma_{h}^{2}}\right) \\
& f_{w}\left(x_{w}, y_{w}\right)=\int_{-\infty}^{\infty} \int_{-\infty}^{\infty} f_{h w}\left(x_{h}, x_{w}, y_{h}, y_{w}\right) \mathrm{d} x_{h} \mathrm{~d} y_{h}=\frac{P}{2 \pi \sigma_{w}^{2}} \exp \left(-\frac{1}{2} \frac{x_{w}^{2}+y_{w}^{2}}{\sigma_{w}^{2}}\right)
\end{aligned}
$$

Fig. 1 shows a section of these marginal distributions. Wilkins' (1969) model, which assumes that commuters select their homes without considering their workplace locations, is a special case of the joint distribution, $f_{h w}$, in which homes and workplaces are independent, i.e., $\rho=0$. In this case, the joint distribution can be written as the product of the two marginal distributions divided by $P$.

In summary, the following three variables are important: (1) $\sigma_{h}$, (2) $\sigma_{w}$, and (3) $\rho$. Let us introduce a parameter $\alpha=\sigma_{w} / \sigma_{h}$ to replace $\sigma_{w}$. Since $\sigma_{h}$ is usually greater than $\sigma_{w}$ (homes are spread over a larger area of the city than are workplaces), we can assume that $0 \leq \alpha \leq 1$.

Fig. 2 shows the change in the distribution of homes for those who work at $x_{w}=1$, with respect to $\rho$ when $\alpha=\sigma_{w} / \sigma_{h}=1 / 2$. Eq. (15) can be rewritten as

$$
\begin{aligned}
& f_{h w}\left(x_{h}, x_{w}, y_{h}, y_{w}\right)=\frac{P}{2 \pi \sigma_{w}^{2}} \exp \left[-\frac{1}{2} \frac{x_{w}^{2}+y_{w}^{2}}{\sigma_{w}^{2}}\right] \\
& \cdot \frac{1}{2 \pi \sigma_{h}^{2}\left(1-\rho^{2}\right)} \exp \left[-\frac{1}{2\left(1-\rho^{2}\right)}\left\{\left(\frac{x_{h}}{\sigma_{h}}-\frac{\rho x_{w}}{\sigma_{w}}\right)^{2}+\left(\frac{y_{h}}{\sigma_{h}}-\frac{\rho y_{w}}{\sigma_{w}}\right)^{2}\right\}\right], \\
&=f_{w}\left(x_{w}, y_{w}\right) f_{h \mid w}\left(x_{h}, y_{h} \mid x_{w}, y_{w}\right)
\end{aligned}
$$

where $f_{h \mid w}\left(x_{h}, y_{h} \mid x_{w}, y_{w}\right)$ is the conditional distribution of homes for workers who work at $\left(x_{w}, y_{w}\right)$. 
A section of the distribution $f_{h \mid w}\left(x_{h}, y_{h} \mid 1,0\right)$ is shown in Fig. 2. If $\rho=0$, i.e., Wilkins' model, the distribution of homes for a given workplace does not depend on the workplace location and coincides with the marginal distribution of homes, the peak of which is at $x=0$. In this case, assignment of a home to a workplace is a random assignment. If $\rho=1$, the locations of homes and workplaces are perfectly correlated, so that all those who work at $x_{w}=1$ live at $x_{h}=2$. We refer to this as minisum assignment because the average commuting distance is minimized with respect to $\rho$, as will be proved later. In contrast, if $\rho=-1$, the locations of homes and workplaces are perfectly correlated, so that all those who work at $x_{w}=1$ live at $x_{h}=-2$, which is located beyond the city center. Thus, all workers must cross the center of the city. In this case, the average commuting distance is maximized with respect to $\rho$, and therefore, we refer to this as maxisum assignment. For a general $\rho$, the distribution of homes has a peak in the interval -2 $<x<2$. In particular, if $\rho=\alpha$, the peak of the distribution of homes at a given workplace coincides with the workplace location. In the example shown in Fig. 2, the distribution of homes for those who work at $x_{w}=1$ has a peak at $x=1$ for $\rho=\alpha=1 / 2$. We refer to this as neighbor assignment.

\subsection{Average commuting distance}

We do not derive the average commuting distance by calculating the mean direct trip length defined by

$$
\begin{aligned}
\bar{d}= & E\left[\sqrt{\left(x_{h}-x_{w}\right)^{2}+\left(y_{h}-y_{w}\right)^{2}}\right] \\
& =\int_{-\infty}^{\infty} \int_{-\infty}^{\infty} \int_{-\infty}^{\infty} \int_{-\infty}^{\infty} \sqrt{\left(x_{h}-x_{w}\right)^{2}+\left(y_{h}-y_{w}\right)^{2}} f_{h w}\left(x_{h}, x_{w}, y_{h}, y_{w}\right) \mathrm{d} x_{h} \mathrm{~d} x_{w} \mathrm{~d} y_{h} \mathrm{~d} y_{w} .
\end{aligned}
$$

Instead, we follow Blumenfeld's (1977) approach and derive the expected value of the commuting distance:

$$
d=\sqrt{\left(x_{h}-x_{w}\right)^{2}+\left(y_{h}-y_{w}\right)^{2}} .
$$

Since each of the four variables, $x_{h}, x_{w}, y_{h}$, and $y_{w}$, has a univariate normal distribution with variances that are equivalent to the spreads of homes or workplaces, we have

$$
\begin{aligned}
& x_{h}, y_{h} \sim N\left(0, \sigma_{h}^{2}\right), \\
& x_{w}, y_{w} \sim N\left(0, \sigma_{w}^{2}\right) .
\end{aligned}
$$

We also have the following relation derived on the basis of the discussion so far:

$$
\operatorname{Cov}\left(x_{h}, x_{w}\right)=\operatorname{Cov}\left(y_{h}, y_{w}\right)=\rho \sigma_{h} \sigma_{w} .
$$

Therefore, the difference in the location of a home and a workplace is also distributed according to a normal distribution, i.e.,

$$
x_{h}-x_{w}, y_{h}-y_{w} \sim N\left(0, \sigma_{h}^{2}+\sigma_{w}^{2}-2 \rho \sigma_{h} \sigma_{w}\right) .
$$

By standardizing the variables, we obtain

$$
\frac{x_{h}-x_{w}}{\sqrt{\sigma_{h}^{2}+\sigma_{w}^{2}-2 \rho \sigma_{h} \sigma_{w}}}, \frac{y_{h}-y_{w}}{\sqrt{\sigma_{h}^{2}+\sigma_{w}^{2}-2 \rho \sigma_{h} \sigma_{w}}} \sim N(0,1) .
$$

The square of $d$ is the sum of the square of two variables. Thus,

$$
\frac{d^{2}}{\sigma_{h}^{2}+\sigma_{w}^{2}-2 \rho \sigma_{h} \sigma_{w}}=\frac{\left(x_{h}-x_{w}\right)^{2}+\left(y_{h}-y_{w}\right)^{2}}{\sigma_{h}^{2}+\sigma_{w}^{2}-2 \rho \sigma_{h} \sigma_{w}} \sim \chi^{2}(2) .
$$

The probability density function of $x$ for the $\chi^{2}$ distribution with two degrees of freedom is $\frac{1}{2} \exp \left(-\frac{x}{2}\right)$. Therefore, the probability density function of $u=d^{2}$ is given by

$$
\frac{1}{2\left(\sigma_{h}^{2}+\sigma_{w}^{2}-2 \rho \sigma_{h} \sigma_{w}\right)} \exp \left(-\frac{u}{2\left(\sigma_{h}^{2}+\sigma_{w}^{2}-2 \rho \sigma_{h} \sigma_{w}\right)}\right) \text {. }
$$


The mean of $d, \bar{d}$, is given by

$$
\begin{aligned}
\bar{d} & =E\left(\sqrt{d^{2}}\right)=\int_{0}^{\infty} \sqrt{u} \frac{1}{2\left(\sigma_{h}^{2}+\sigma_{w}^{2}-2 \rho \sigma_{h} \sigma_{w}\right)} \exp \left(-\frac{u}{2\left(\sigma_{h}^{2}+\sigma_{w}^{2}-2 \rho \sigma_{h} \sigma_{w}\right)}\right) \mathrm{d} u \\
& =\sqrt{2} \sqrt{\sigma_{h}^{2}+\sigma_{w}^{2}-2 \rho \sigma_{h} \sigma_{w}} \Gamma\left(\frac{3}{2}\right)=\sqrt{\frac{\pi}{2}\left(\sigma_{h}^{2}+\sigma_{w}^{2}-2 \rho \sigma_{h} \sigma_{w}\right)},
\end{aligned}
$$

which indicates that the average commuting distance is explained by the previously mentioned three parameters. Using $\alpha=\sigma_{w} / \sigma_{h}$ instead of $\sigma_{w}$, we can rewrite (28) as

$$
\bar{d}=\sigma_{h} \sqrt{\frac{\pi}{2}\left(1+\alpha^{2}-2 \rho \alpha\right)} .
$$

\subsection{Urban structure and average commuting distance}

The parameter $\alpha=\sigma_{w} / \sigma_{h}$ represents the difference not only between the spreads of homes and workplaces but also between the densities of homes and workplaces in the city center, i.e.,

$$
\frac{f_{h}(0,0)}{f_{w}(0,0)}=\frac{\sigma_{w}^{2}}{\sigma_{h}^{2}}=\alpha^{2},
$$

which can be called jobs-housing balance. Usually, $\sigma_{h}$ is larger than $\sigma_{w}$, and hence, we can assume that $0 \leq \alpha \leq 1$. If $\alpha$ is close to $1, \sigma_{h}$ and $\sigma_{w}$ are almost equal, and jobs-housing balance is achieved in an employment-decentralized (dispersed) urban structure. If $\alpha$ is small, the difference between $\sigma_{h}$ and $\sigma_{w}$ is large, and employment concentration in the city center destroys the jobs-housing balance. In this study, the parameter $\alpha$ is used to determine the urban structure. Then, Eq. (29) implies that $\bar{d}$ is determined by the following three parameters: $\sigma_{h}, \rho$, and the jobs-housing balance $\alpha . \sigma_{h}$ has a simple proportional effect on $\bar{d}$, and hence, we focus on the effects of the other two parameters.

Fig. 3 shows how $\bar{d}$ varies with $\alpha$ and $\rho$; this plot is called Brotchie's urban triangle. From this figure, we observe the following:

i) If jobs are completely concentrated, i.e., $\alpha=0$, the average distance is given by

$$
\bar{d}=\sqrt{\frac{\pi}{2}} \sigma_{h},
$$

and the same distance is achieved if $\alpha=2 \rho . \bar{d}$ becomes smaller than this criterion only if $\alpha<2 \rho$ (intense concentration or strong correlation).

ii) If jobs are perfectly dispersed (implies that $\alpha=1$ ) and fully correlated (implies that $\rho=1$ ), the locations of homes and workplaces perfectly coincide and commuting disappears, i.e., $\bar{d}=0$.

iii) If $\rho$ is known, the parameter $\alpha$, which contributes to the minimization of $\bar{d}$, is found by solving the following first-order condition of the rooted term of Eq. (29):

$$
\frac{\partial}{\partial \alpha}\left(1+\alpha^{2}-2 \rho \alpha\right)=2(\alpha-\rho)=0 .
$$

Thus, we have

$$
\alpha=\rho .
$$

If $\rho$ is given a priori, we can control $\alpha$ on the basis of some land-use policies. If $\alpha>\rho$, we obtain $\partial \bar{d} / \partial \alpha>0$, and the average commuting distance increases owing to workplace decentralization. On the other hand, if $\alpha<\rho$, we obtain $\partial \bar{d} / \partial \alpha<0$, and the average distance decreases owing to workplace decentralization. 
3.5 Average distance for typical correlations of homes and workplaces

Recall the four typical types of home-workplace assignment defined in Section 3.2: random, minisum, maxisum, and neighbor assignment. We will calculate the average commuting distances for these assignments.

\subsubsection{Random assignment}

Random assignment is the case where $\rho=0$ and workers choose their workplace randomly without considering the location of their homes. This assignment brings about a random commute. The random average commute distance is given by

$$
\bar{d}_{\text {rand }}=\sqrt{\frac{\pi}{2}\left(\sigma_{h}^{2}+\sigma_{w}^{2}\right)}=\sigma_{h} \sqrt{\frac{\pi}{2}\left(1+\alpha^{2}\right)} \text {. }
$$

\subsubsection{Minisum assignment}

The full correlation, $\rho=1$, between homes and workplaces minimizes the total commuting distance ( $\alpha$ is constant in this case), and hence, brings about a minimum average commute. The minimum average commute distance is given by

$$
\bar{d}_{\min }=\sqrt{\frac{\pi}{2}}\left(\sigma_{h}-\sigma_{w}\right)=\sigma_{h} \sqrt{\frac{\pi}{2}}(1-\alpha) .
$$

\subsubsection{Maxisum assignment}

The full negative correlation, $\rho=-1$, between homes and workplaces maximizes the total commuting distance ( $\alpha$ is constant), and hence, brings about a maximum average commute. The maximum average commute distance is given by

$$
\bar{d}_{\max }=\sqrt{\frac{\pi}{2}}\left(\sigma_{h}+\sigma_{w}\right)=\sigma_{h} \sqrt{\frac{\pi}{2}}(1+\alpha) .
$$

\subsubsection{Neighbor assignment}

In the case of $\alpha=\rho$, the average commuting distance is minimized for a given $\rho$ and is obtained by

$$
\bar{d}_{n e i}=\sqrt{\frac{\pi}{2}\left(\sigma_{h}^{2}-\sigma_{w}^{2}\right)}=\sigma_{h} \sqrt{\frac{\pi}{2}\left(1-\alpha^{2}\right)} .
$$

This should be close to the real-world commuting pattern because the assignment pattern has a peak in the conditional distribution of homes at the corresponding workplaces. Thus, we call this neighbor average commute distance and see it as an estimate of the observed average commute distance.

If we can know two of these average distances, we can obtain estimates of $\sigma_{h}$ and $\sigma_{w}$, and, thus, obtain jobs-housing balance $\alpha$. Then we can also obtain the estimate of spatial correlation $\rho$ by using Eq. (29).

The functional forms of the average commuting distances for the four typical assignment patterns with respect to changes in $\alpha$ are shown in Fig. 4. The average distance under random assignment increases as the jobs-housing relation becomes balanced and decreases as the jobs are dispersed under minisum assignment. The average distance under 
neighbor assignment decreases to a small extent as the urban structure becomes balanced.

\subsection{Jobs-housing imbalance and excess commuting}

Excess commuting is defined as the difference between the observed average and the minimum average commute distance or time. It is usually presented as the excess ratio (called excess hereafter), $E_{c}$, which is defined as the ratio of the aforementioned difference and the observed average commute. Therefore,

$$
E_{c}=\frac{\bar{d}-\bar{d}_{\min }}{\bar{d}}=1-\frac{\sigma_{h}-\sigma_{w}}{\sqrt{\sigma_{h}^{2}+\sigma_{w}^{2}-2 \rho \sigma_{h} \sigma_{w}}}=1-\frac{1-\alpha}{\sqrt{1+\alpha^{2}-2 \rho \alpha}},
$$

which implies that the excess can be expressed by $\alpha$ and $\rho$ (the scale variable $\sigma_{h}$ has been eliminated).

Fig. 5 shows the relation among the three variables: spatial correlation, jobs-housing balance, and excess commuting. We observe the following:

i) If $\alpha=0$, i.e., workplaces are perfectly centralized, no excess exists. At the other extreme, if $\alpha=1$, i.e., the distributions of homes and workplaces coincide, all commutes have excess.

ii) The excess decreases as $\alpha$ decreases. In other words, a highly job centralized city has less excess commuting.

iii) The excess decreases as $\rho$ increases. In other words, a city in which jobs and housing are highly positively correlated and sectorially structured has less excess commuting.

We cannot say what the actual assignment pattern of homes and workplaces is, because this pattern is complicated in practice. However, from a macroscopic view, we can regard neighbor assignment as the actual assignment pattern. This is because for almost all cities, the inner commute is dominant and a greater number of workers live close to the city center than away from the center. Therefore, we take the neighbor assignment to be the actual assignment.

By considering $\rho=\alpha$, we express the excess as

$$
E_{c}=\frac{\bar{d}_{n e i}-\bar{d}_{\min }}{\bar{d}_{n e i}}=1-\frac{1-\alpha}{\sqrt{1-\alpha^{2}}},
$$

where $\alpha$ is the only variable. Excess commuting under the neighbor assignment is explained by the jobs-housing balance, as shown in Fig. 5. This graph shows that the greater the dispersion of jobs, the higher is the excess. This explains the difference in the excess between US and Japanese/Korean cities.

\subsection{Jobs-housing imbalance and capacity utilization}

Capacity utilization, $C_{u}$, is defined as the usage of the available commuting range, given a fixed spatial distribution of residences and workplaces. This value is useful for comparing the commuting efficiency of different cities. $C_{u}$ is calculated by dividing the excess commuting by the total commuting capacity, i.e., the difference between the maximum and minimum commutes (Charron, 2007; Horner, 2002; Murphy, 2009). Therefore,

$$
\begin{aligned}
C_{u} & =\frac{\bar{d}-\bar{d}_{\min }}{\bar{d}_{\max }-\bar{d}_{\min }}=\frac{\sqrt{\sigma_{h}^{2}+\sigma_{w}^{2}-2 \rho \sigma_{h} \sigma_{w}}-\left(\sigma_{h}-\sigma_{w}\right)}{\left(\sigma_{h}+\sigma_{w}\right)-\left(\sigma_{h}-\sigma_{w}\right)} \\
& =\frac{\sqrt{1+\alpha^{2}-2 \rho \alpha}-(1-\alpha)}{2 \alpha},
\end{aligned}
$$

which implies that $C_{u}$ can be expressed in terms of the variables $\alpha$ and $\rho$. 
Fig. 6 shows the relation among the three variables: spatial correlation, jobs-housing balance, and capacity utilization. We observe the following:

i) If $\rho=1$, i.e., homes and workplaces are perfectly positively correlated (commuting distance is minimized), no commuting capacity is used. At the other extreme, if $\rho=-1$, i.e., homes and workplaces are perfectly negatively correlated (commuting distance is maximized), all the commuting capacity is utilized.

ii) The $C_{u}$ decreases as the jobs-housing balance, $\alpha$, decreases. In other words, a highly job centralized city has less capacity utilization.

iii) The $C_{u}$ decreases as $\rho$ increases. In other words, a city in which jobs and housing are highly positively correlated and sectorially structured has less capacity utilization. By considering $\rho=\alpha$, we express the $C_{u}$ as

$$
C_{u}=\frac{\bar{d}_{n e i}-\bar{d}_{\min }}{\bar{d}_{\max }-\bar{d}_{\min }}=\frac{\sqrt{1-\alpha^{2}}-(1-\alpha)}{2 \alpha},
$$

where $\alpha$ is the only variable. The $C_{u}$ under the neighbor assignment is explained by the jobs-housing balance, as shown in Fig. 6 . This graph shows that the smaller the $C_{u}$, the larger is the number of jobs dispersed.

\subsection{Commuting economy and normalized commuting economy}

Commuting economy, $C_{e}$, is defined as the extent to which the observed average commute is falling below or above the random average commute - that is, the extent to which collective behavior as expressed by the observed trip pattern is departing from random behavior and reacting to the consumption of zonal separation (Murphy and Killen, 2011). $C_{e}$ is calculated by

$$
C_{e}=1-\frac{\bar{d}}{\bar{d}_{\text {rand }}}=1-\frac{\sqrt{\sigma_{h}^{2}+\sigma_{w}^{2}-2 \rho \sigma_{h} \sigma_{w}}}{\sqrt{\sigma_{h}^{2}+\sigma_{w}^{2}}}=1-\frac{\sqrt{1+\alpha^{2}-2 \rho \alpha}}{\sqrt{1+\alpha^{2}}},
$$

which implies that $C_{u}$ can be expressed in terms of the variables $\alpha$ and $\rho$. By considering $\rho=\alpha$, we express the $C_{e}$ as

$$
C_{e}=1-\frac{\bar{d}_{n e i}}{\bar{d}_{\text {rand }}}=1-\frac{\sqrt{1-\alpha^{2}}}{\sqrt{1+\alpha^{2}}},
$$

where $\alpha$ is the only variable.

Normalized commuting economy, $N C_{e}$, is the extent to which the observed average commute is below the random average commute relative to the minimum average commute, given a fixed spatial distribution of residences and workplaces (Murphy and Killen, 2011). This value is calculated by

$$
\begin{aligned}
N C_{e} & =\frac{\bar{d}_{\text {rand }}-\bar{d}}{\bar{d}_{\text {rand }}-\bar{d}_{\min }}=\frac{\sqrt{\sigma_{h}^{2}+\sigma_{w}^{2}}-\sqrt{\sigma_{h}^{2}+\sigma_{w}^{2}-2 \rho \sigma_{h} \sigma_{w}}}{\sqrt{\sigma_{h}^{2}+\sigma_{w}^{2}}-\left(\sigma_{h}-\sigma_{w}\right)} \\
& =\frac{\sqrt{1+\alpha^{2}}-\sqrt{1+\alpha^{2}-2 \rho \alpha}}{\sqrt{1+\alpha^{2}}-(1-\alpha)},
\end{aligned}
$$

which implies that $N C_{e}$ also can be expressed in terms of the variables $\alpha$ and $\rho$. By considering $\rho=\alpha$, we express the $N C_{e}$ as

$$
N C_{e}=\frac{\bar{d}_{\text {rand }}-\bar{d}_{\text {nei }}}{\bar{d}_{\text {rand }}-\bar{d}_{\text {min }}}=\frac{\sqrt{1+\alpha^{2}}-\sqrt{1-\alpha^{2}}}{\sqrt{1+\alpha^{2}}-(1-\alpha)},
$$

where $\alpha$ is the only variable. 


\section{Discussion}

We have explained the relationship between urban structure and commuting distance explicitly and theoretically. We used Vaughan's (1974) model to calculate the unbiased excess commuting using a continuous urban structure instead of a zonal model.

The average commuting distance is clearly described by the jobs-housing balance and the spatial correlation, and this can explain many results discussed in previous literatures. The degree of decentralization of workplaces can contribute to an increase or a decrease in the average commuting distance depending on the spatial correlation, as has already been observed by Ma and Banister (2007). Van Ommeren et al. (1996) found that the residence and workplace locations can be jointly determined and that the residential mobility and labor market mobility are mutually dependent. They used a bivariate duration model of residential and labor market mobility and took into account commuting costs, with the aim of developing a simultaneous search model for labor and housing markets. Their empirical results showed that residential mobility and labor mobility are positively related. Thus, it is natural that we consider the locations of homes and workplaces to be interrelated.

From the discussion in Section 3.5, if we assume that the actual home-workplace assignment is neighbor assignment, we can decrease the commuting distance by decentralization of workplaces. However, the decrease in this case is not large. If we can control $\rho$ by adopting policies such as activation of the housing market, encouraging minisum assignment should be an effective way to reduce commuting time.

How can we interpret the policies promoting the decentralization of workplaces, such as those promoted by the Japanese Government for the Tokyo region? If workplaces are already dispersed to some extent $(\alpha>\rho)$, the commuting distance will increase as a result of the decentralization policy. In contrast, if workplaces are concentrated $(\alpha<\rho)$, the commuting distance will decrease. The urban structure in the Tokyo region seems to correspond to the latter case, and therefore, the decentralization policy is justified.

We verified that the excess commuting, capacity utilization, and commuting economy can be expressed by the jobs-housing balance and the spatial correlation. Excess commuting is also an important measure of the appropriateness of the urban structure. We found that if the actual home-workplace assignment is close to neighbor assignment, excess commuting increases when the decentralization of workplaces is promoted. This implies that the potential for decreasing the commuting distance could be large when a decentralization policy is implemented. Combining this with a policy for the promotion of home-workplace matching appears to be a powerful option for shortening commutes.

It is expected that $\rho$ would increase with the city size since in a small city, every home is almost equally accessible from every workplace. In a large city, however, workplaces on the same side of the city as the worker's home will be more accessible than those on the other side of the city. Vaughan (1987) presented estimated values of $\alpha$ and $\rho$ for UK and Australian cities: $\alpha=0.6$ and $\rho=0.2$ in small cities, and $\alpha=\rho=0.8$ in large cities.

Although we do not have a precise estimate, the Tokyo metropolitan area seems to have a much smaller $\alpha$ and a much larger $\rho$. If this is true, our theory predicts that decentralization contributes to a decrease in the commuting distance and that the excess takes smaller values. This is consistent with the observations in Section 2 of this paper and those in Merriman et al. (1995).

Giuliano and Small (1993, p.1496) concluded that "attempts to alter the metropolitan-wide structure of urban land use via policy intervention are likely to have disappointing impacts on commuting patterns, even if successful in changing the degree of jobs-housing balance." Figs. 3 and 4 show that the average commuting distance is not affected by changes in $\alpha$ or $\rho$. Generally, since the growth of a city increases the spread of homes 
and nullifies the decrease in $\bar{d}$, the average commuting distance does not change drastically. Our model explains this to some extent and supports the results proposed by Giuliano and Small (1993).

We clarified the positioning of cities by calculating the minimum and maximum commutes, in order to compare the relationship between the commuting efficiency and urban structure of different cities using two indices: excess commuting and capacity utilization.

Fig. 7 presents the theoretical relationship between excess commuting and capacity utilization, and Fig. 8 shows the empirical relationship between the two indices in selected cities in the US and in Japan/Korea. The excess commuting and capacity utilization of the US cities are obtained from Horner (2002), and those for the Japanese/Korean cities are obtained from Lee et al. (2006). Table 1 gives the commuting distance, excess commuting, and capacity utilization obtained from these papers.

As Fig. 8 shows, both excess commuting and capacity utilization are higher in the US cities than in the Japanese/Korean cities. However, there is no significant difference between the excess and $C_{u}$ values in the Japanese and Korean cities. The results show that the urban spatial structure - the distribution of homes and workplaces - is more dispersed in US cities than in Japanese and Korean cities. Further, the commuting inefficiency is higher in the US cities than in the Japanese/Korean cities. The capacity utilization values, too, are high for the US cities. Murphy's (2009) claim that excess commuting is considerably greater for private transport than for public transport is also supported. The high commuting inefficiency in the US cities is because of the fact that the use of private transport is more common in the US than in Japan/Korea.

The model presented in this paper still cannot cope with milticentric workers distribution because Vaughan's model has only one peak at the center. Therefore, the fitness to the model may be relatively low in cities that have multiple business centers. However, the introduction of spatial correlation allows us to widen the applicability of continuous modeling to the discussion on commuting efficiency. Especially, explicit expressions of several typical jobs-housing assignment ways are powerful in understanding the relationship between commuting and urban spatial structure, the concept of which is presented by Brotchie (1984).

\section{Conclusions}

This study shows that the relationship between urban structure, i.e., the distributions of homes and workplaces, and three indices-average commuting distance, excess commuting, and capacity utilization - can be theoretically analyzed by using two variables: the jobs-housing balance and the spatial correlation.

The results can be summarized as follows. First, decentralization of workplaces can cause an increase or a decrease in the average commuting distance depending on the spatial correlation. However, to decrease the commuting distance when neighbor assignment is assumed, the urban structure should become balanced to some extent. Moreover, the commuting distance is zero in the case of minisum assignment, i.e., if jobs are perfectly dispersed and fully correlated with homes. Thus, controlling the jobs-housing balance or the spatial correlation could be an effective way to reduce the commuting distance.

Second, excess commuting increases when decentralization of workplaces is promoted. This implies that the potential for decreasing the commuting distance could be large when a decentralization policy is implemented. This may help explain the differences in the urban structure among different cities.

Third, care should be taken when interpreting the efficiency of the urban structure of different cities using excess commuting, because the urban structure and commuting pattern are different for different cities, as observed by Ma and Banister (2007). Thus, both indices, i.e., 
excess commuting and capacity utilization, are necessary to compare the commuting efficiency and urban structure of different cities.

Finally, we suggested comparing the different urban structures of US and Japanese/Korean cities using excess commuting and capacity utilization. The excess is larger for US cities, but the commuting efficiency is poorer than that in Japanese or Korean cities; this is because US cities generally have a more dispersed and automobile-dependent urban structure than do Japanese or Korean cities.

It is worthy that the theoretical modeling approach using continuous distribution is still has a possibility to draw a more realistic commuting structure by introducing spatial correlation between homes and workplaces. Several typical jobs-housing assignment ways enables us to express explicitly the conceptual notion of the relationship between commuting and urban spatial structure.

Since the model proposed here is also a monocentric model as Hamilton's one, however, there are limitation to fit the actual urban spatial structure that often has multicentric patterns. The accuracy and limitation of the model need to be investigated by measuring spatial distribution of homes and workplaces, jobs-housing balance, spatial correlation between homes and workplaces, and benchmark measures of commuting efficiency of real-world cities. We compared some data for US and Japanese/Korean cities, but the number of samples is still limited. To measure the three key parameters mentioned in this study from actual commuting data, to analyze the effects of job decentralization policies, and to carry out model studies on the basis of multicentric continuous distribution are the remaining research agenda for the future.

\section{References}

Alonso, W., 1964. Location and Land Use. Harvard University Press, Cambridge, Mass.

Angel, S., Hyman, G.M., 1972. Urban Spatial Interaction. Environment and Planning A 4, 99-118.

Black, J.A., Katakos, A., 1987. Optimisation Methods and the Classification of City Structure: Theory and Empirical Testing. Environment and Planning B 14, 93-107.

Blumenfeld, D.E., 1977. Modeling the Joint Distribution of Home and Workplace Locations in a City. Transportation Science 11(4), 307-337.

Blumenfeld, D.E., Weiss, G.H., 1974. Some Radial and Direction-Dependent Models for Densities of Homes and Workplaces. Transportation Research 8, 149-155.

Boussauw, K., Derudder, B., Witlox, F., 2011. Measuring Spatial Separation Processes through the Minimum Commute: The Case of Flanders. European Journal of Transport and Infrastructure Research 11(1), 42-60.

Brotchie, J.F., 1984. Technological Change and Urban Form. Environment and Planning A 16, 583-596.

Brotchie, J.F., Anderson, M., Gipps, P.G., McNamara, C., 1996. Urban Productivity and Sustainability - Impacts of Technological Change. In Y. Hayashi and J. Roy (eds.), Transport, Land-Use and the Environment, Kluwer Academic Publishers, Netherlands, 81-99.

Charron, M., 2007. From Excess Commuting to Commuting Possibilities: More Extension to the Concept of Excess Commuting. Environment and Planning B 39, 1238-1254.

Clark, C., 1951. Urban Population Densities. Journal of the Royal Statistical Society 114, 490-496.

Fairthorne, D., 1965. The Distances Between Pairs of Points in Towns of Simple Geometrical Shapes. In J. Almond (ed.), Proceedings of the $2^{\text {nd }}$ International Symposium on the Theory of Traffic Flow, London, 1963, OECD, Paris, 391-406.

Frost, M., Linneker, B., Spence, N., 1998. Excess or Wasteful Commuting in a Selection of British Cities. Transportation Research Part A 32(7), 529-538. 
Giuliano, G., Small, K.A., 1993. Is the Journey to Work Explained by Urban Structure? Urban Studies 30(9), 1485-1500.

Haight, F.A., 1964. Some Probability Distributions Associated with Commuter Travel in a Homogeneous Circular City. Operations Research 12, 964-975.

Hamilton, B.W., 1982. Wasteful Commuting. Journal of Political Economy 90(5), 1035-1053.

Hamilton, B.W., 1989. Wasteful Commuting Again. Journal of Political Economy 97(6), 1497-1504.

Horner, M.W., 2002. Extensions to the Concept of Excess Commuting. Environment and Planning B 34, 543-566.

Horner, M.W., 2004. Spatial Dimensions of Urban Commuting: A Review of Major Issues and Their Implications for Future Geographic Research. The Professional Geographer 56(2), 160-173.

Horner, M.W., 2010. Exploring the Sensitivity of Jobs-Housing Statistics to Imperfect Travel Time Information. Environment and Planning B 37, 367-375.

Horner, M.W., 2010. How Does Ignoring Worker Class Impact Measuring Jobs-housing Balance? An Exploratory Spatial Data Analysis. Transportation Research Board 2163, 57-64.

Horner, M.W., Murray, A.T., 2002. Excess Commuting and the Modifiable Areal Unit Problem. Urban Studies 39(1), 131-139.

Kim, S., 1995. Excess Commuting for Two-Worker Households in the Los Angeles Metropolitan Area. Journal of Urban Economics 38, 166-182.

Layman, C.C., Horner, M.W., 2010. Comparing Methods for Measuring Excess Commuting and Jobs-housing Balance: An Empirical Analysis of Land Use Changes. Transportation Research Board 2174, 110-117.

Lee, S.H., Suzuki, T., Lee, M.H., 2006. A Study on the Change of Urban Structure and Commuting Based on Optimal Commuting Assignment Problem in Korean and Japanese Metropolitan Areas. Journal of the Korea Planners Association 41(2), 57-65 (in Korean).

Ma, K.R., Banister, D., 2006a. Excess Commuting: A Critical Review. Transport Reviews 26(6), 749-767.

Ma, K.R., Banister, D., 2006b. Extended Excess Commuting: A Measure of the Jobs-Housing Imbalance in Seoul. Urban Studies 43(11), 2099-2113.

Ma, K.R., Banister, D., 2007. Urban Spatial Change and Excess Commuting. Environment and Planning B 39, 630-646.

Merriman, D., Ohkawara, T., Suzuki, T., 1995. Excess Commuting in the Tokyo Metropolitan Area: Measurement and Policy Simulations. Urban Studies 32(1), 69-85.

Murphy, E., 2009. Excess Commuting and Modal Choice. Transportation Research Part A 43, 735-743.

Murphy, E., Killen E.J., 2011. Commuting Economy: An Alternative Approach for Assessing Regional Commuting Efficiency. Urban Studies 48(6), 1255-1272.

Niedzielski, M.A., 2006. A Spatially Disaggregated Approach to Commuting Efficiency. Urban Studies 43(13), 2485-2502.

O'Kelly, M.E., Lee, W., 2005. Disaggregate Journey-to-Work Data: Implications for Excess Commuting and Jobs-Housing Balance. Environment and Planning A 37, 2233-2252.

Small, K.A., Song, S., 1992. 'Wasteful' Commuting: A Resolution. Journal of Political Economy 100(5), 888-898.

van Ommeren, J., Rietveld, P., Nijkamp, P., 1996. Residence and Workplace Relocation: A Bivariate Duration Model Approach. Geographical Analysis 28(4), 315-329.

Vaughan, R., 1974. Traffic Activity Described in Terms of Some Characteristics of an Urban Area. Transportation Research 8, 553-565.

Vaughan, R., 1987. Urban Spatial Traffic Patterns. Pion Ltd., London.

White, M.J., 1988. Urban Commuting Journeys are not 'Wasteful.' Journal of Political 
Economy 96(5), 1097-1110.

Wilkins, C.A., 1969. Sherratt's Model and Commuter Travel. Transportation Science 3, 93-98. 
Table 1. Average commuting distance, excess commuting, and capacity utilization in US and Japanese/Korean cities

\begin{tabular}{|c|c|c|c|c|c|c|c|c|}
\hline \multirow[t]{2}{*}{ Source } & \multirow[t]{2}{*}{ City Name } & \multirow[t]{2}{*}{ Year } & \multirow[t]{2}{*}{$\begin{array}{l}\text { Number of } \\
\text { work trips }\end{array}$} & \multicolumn{3}{|c|}{$\begin{array}{c}\text { Average } \\
\text { Commuting Distance }(\mathrm{km})\end{array}$} & \multirow[t]{2}{*}{$\begin{array}{c}\text { Excess } \\
\text { Commuting }\end{array}$} & \multirow[t]{2}{*}{$\begin{array}{l}\text { Capacity } \\
\text { Utilization }\end{array}$} \\
\hline & & & & Min. & Observed & Max. & & \\
\hline \multirow{27}{*}{$\begin{array}{l}\text { Horner } \\
(2002)\end{array}$} & US cities & \multirow{27}{*}{1990} & & & & & & \\
\hline & Atlanta & & $1,279,104$ & 7.64 & 16.77 & 38.77 & 0.544 & 0.293 \\
\hline & Baltimore & & $1,022,450$ & 4.83 & 12.86 & 32.94 & 0.624 & 0.286 \\
\hline & Boise & & 87,382 & 3.48 & 6.68 & 10.07 & 0.481 & 0.486 \\
\hline & Boston & & $1,946,133$ & 4.72 & 12.15 & 41.96 & 0.612 & 0.200 \\
\hline & Charlotte & & 423,873 & 6.58 & 12.38 & 37.85 & 0.468 & 0.185 \\
\hline & Cincinnati & & 684,950 & 5.02 & 11.96 & 29.77 & 0.580 & 0.280 \\
\hline & Cleveland & & 886,944 & 4.86 & 11.94 & 38.24 & 0.593 & 0.212 \\
\hline & Columbus & & 563,061 & 5.33 & 11.83 & 25.93 & 0.550 & 0.316 \\
\hline & Denver & & 941,325 & 4.63 & 12.28 & 35.73 & 0.622 & 0.246 \\
\hline & Las Vegas & & 356,452 & 4.10 & 10.14 & 18.06 & 0.596 & 0.433 \\
\hline & Memphis & & 360,631 & 3.73 & 11.01 & 20.29 & 0.660 & 0.439 \\
\hline & Miami & & 826,175 & 5.63 & 11.84 & 23.64 & 0.525 & 0.345 \\
\hline & Milwaukee & & 775,000 & 3.80 & 10.65 & 37.19 & 0.644 & 0.205 \\
\hline & Minneapolis/St Paul & & $1,221,765$ & 5.44 & 13.00 & 33.92 & 0.582 & 0.266 \\
\hline & Omaha & & 274,058 & 2.98 & 8.27 & 16.61 & 0.640 & 0.389 \\
\hline & Philadelphia & & $2,133,136$ & 3.80 & 11.60 & 42.23 & 0.672 & 0.203 \\
\hline & Phoenix & & 919,386 & 5.21 & 12.76 & 29.31 & 0.591 & 0.313 \\
\hline & Pittsburgh & & 832,049 & 5.31 & 11.25 & 37.93 & 0.527 & 0.182 \\
\hline & Portland & & 687,845 & 5.75 & 11.65 & 40.41 & 0.506 & 0.170 \\
\hline & Rochester & & 395,118 & 6.08 & 11.81 & 23.71 & 0.485 & 0.325 \\
\hline & Sacramento & & 595,168 & 6.15 & 12.65 & 32.12 & 0.514 & 0.250 \\
\hline & San Antonio & & 506,666 & 4.52 & 12.02 & 21.53 & 0.624 & 0.441 \\
\hline & San Diego & & $1,126,712$ & 4.88 & 14.55 & 40.28 & 0.665 & 0.273 \\
\hline & Seattle & & 1,156,219 & 6.60 & 13.79 & 44.37 & 0.522 & 0.190 \\
\hline & St Louis & & $1,026,857$ & 6.41 & 14.18 & 35.49 & 0.549 & 0.268 \\
\hline & Wichita & & 198,394 & 4.18 & 9.64 & 16.00 & 0.567 & 0.463 \\
\hline \multirow{14}{*}{$\begin{array}{l}\text { Lee et } \\
\text { al. } \\
(2006)\end{array}$} & Japanese cities & & & & & & & \\
\hline & Tokyo & \multirow{7}{*}{2000} & $17,261,325$ & 6.71 & 11.02 & 50.49 & 0.391 & 0.098 \\
\hline & Sapporo & & 1,153,767 & 6.23 & 7.86 & 19.82 & 0.206 & 0.119 \\
\hline & Sendai & & $1,043,212$ & 6.88 & 8.99 & 34.91 & 0.235 & 0.076 \\
\hline & Nagoya & & $4,466,042$ & 4.99 & 8.04 & 40.97 & 0.379 & 0.085 \\
\hline & Osaka & & $8,636,059$ & 5.59 & 9.30 & 50.72 & 0.399 & 0.082 \\
\hline & Hiroshima & & 993,002 & 5.45 & 7.47 & 24.05 & 0.271 & 0.109 \\
\hline & Kitakyusyu/Fukuoka & & $2,457,008$ & 5.18 & 7.69 & 54.38 & 0.326 & 0.051 \\
\hline & Korean cities & & $\begin{array}{c}* 2 \% \\
\text { sample }\end{array}$ & & & & & \\
\hline & Seoul & \multirow{5}{*}{2000} & 154,833 & 5.79 & 9.88 & 37.53 & 0.414 & 0.129 \\
\hline & Busan & & 32,713 & 6.22 & 8.80 & 34.03 & 0.293 & 0.093 \\
\hline & Daegu & & 20,410 & 6.26 & 8.30 & 25.39 & 0.245 & 0.106 \\
\hline & Daejeon & & 16,733 & 6.50 & 8.56 & 38.61 & 0.240 & 0.064 \\
\hline & Gwangju & & 10,768 & 6.85 & 8.38 & 21.54 & 0.182 & 0.104 \\
\hline
\end{tabular}




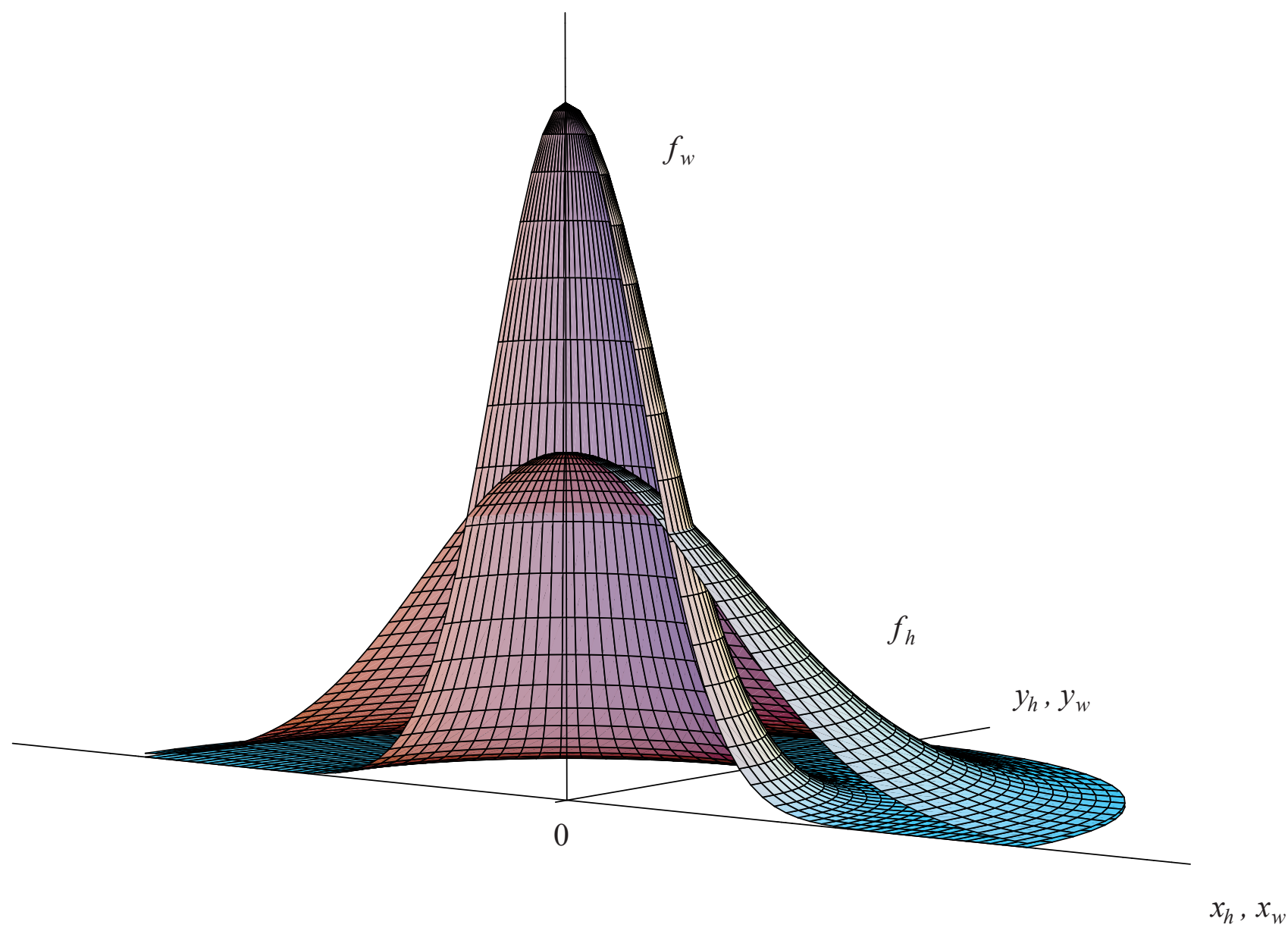

Figure 1. Marginal distributions of homes and workplaces of the quadrivariate joint distribution model. 


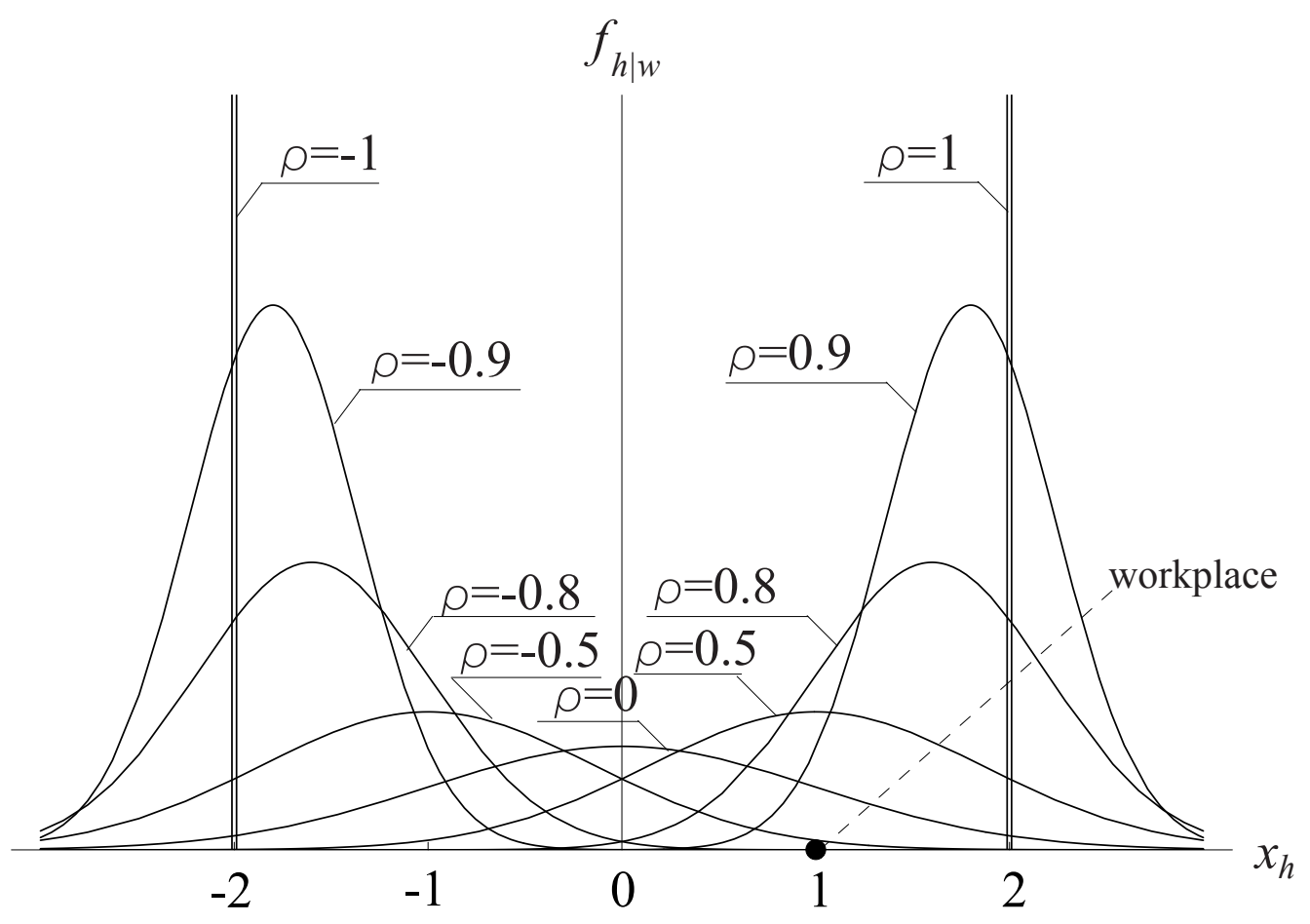

Figure 2. Spatial correlation, $\rho$, and the conditional distributions of homes for those who work at $x_{w}=1$ (in the case of $\alpha=\sigma_{w} / \sigma_{h}=1 / 2$ ). 


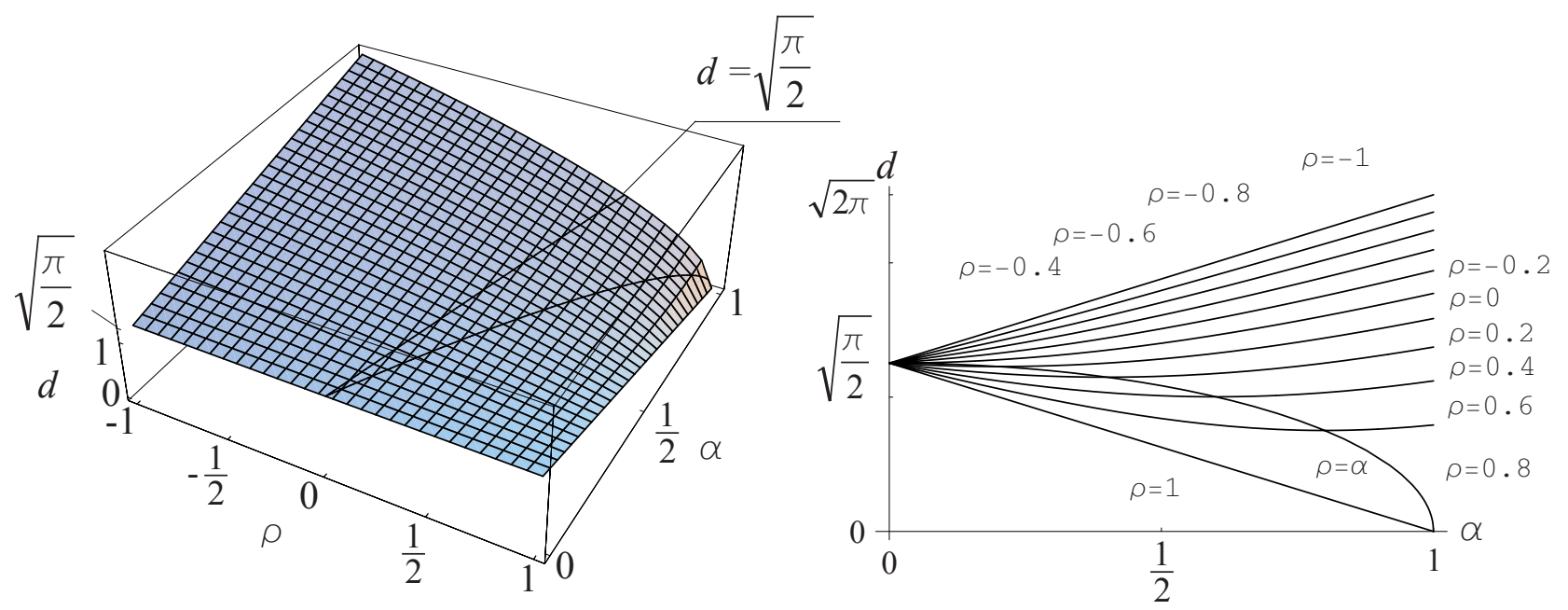

Figure 3. Spatial correlation, jobs-housing balance, and average commuting distance. 


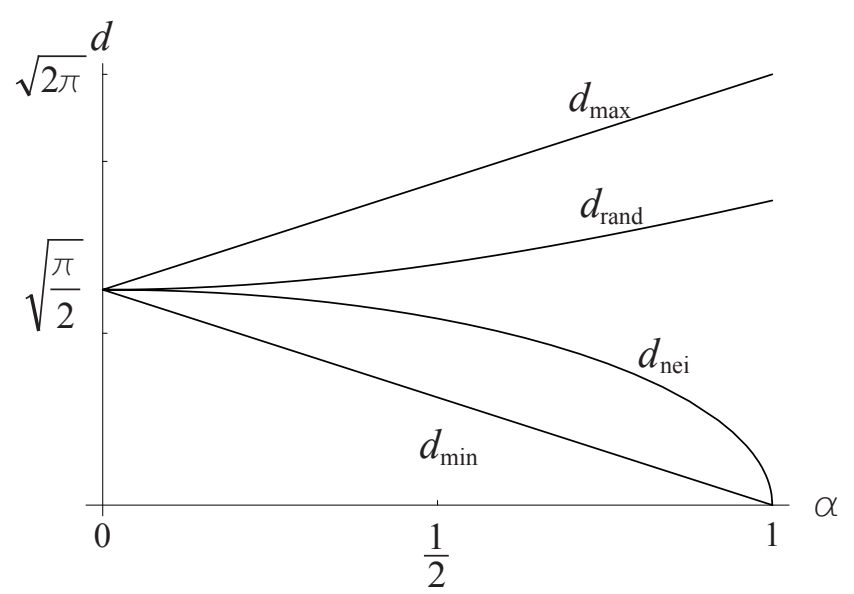

Figure 4. Average commuting distance under four typical assignment patterns. 

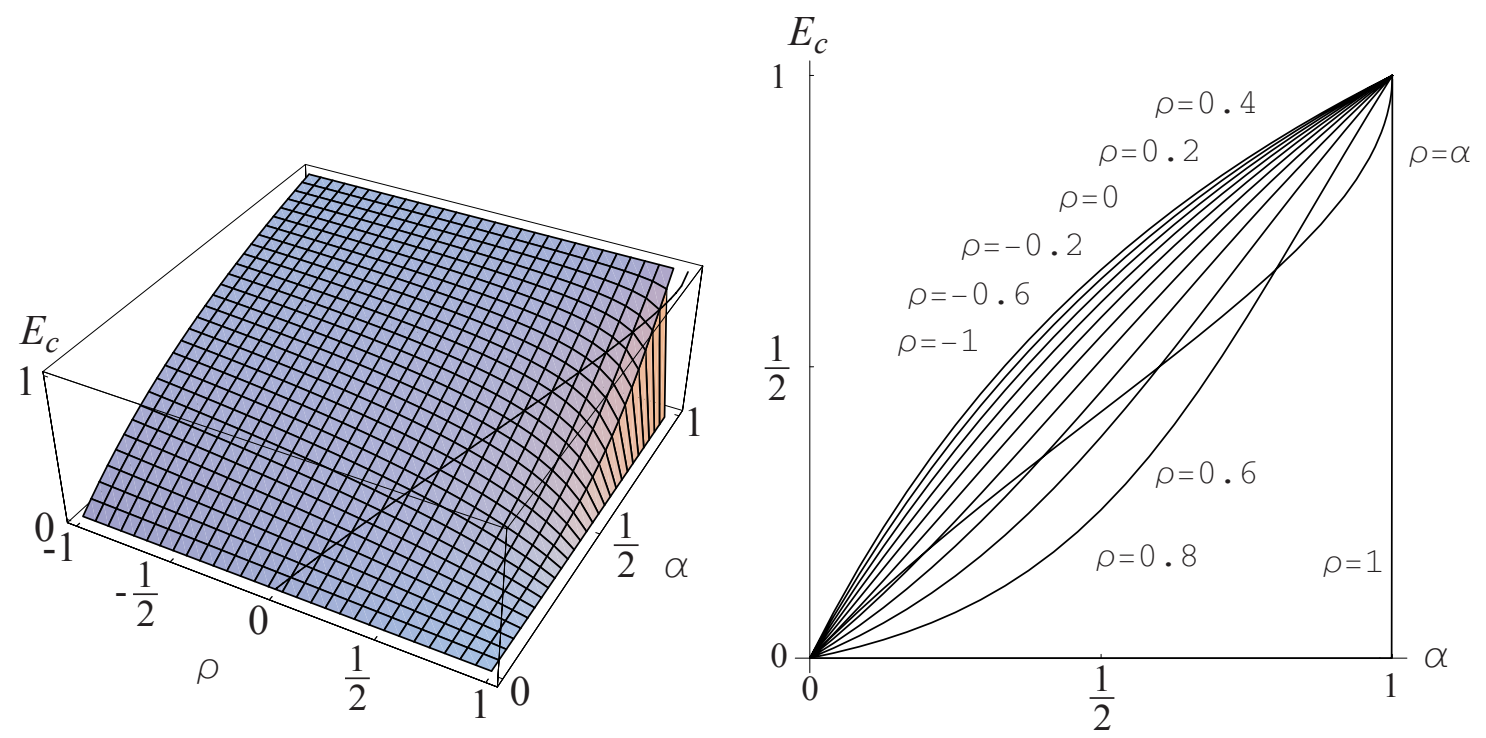

Figure 5. Spatial correlation, jobs-housing balance, and excess commuting. 

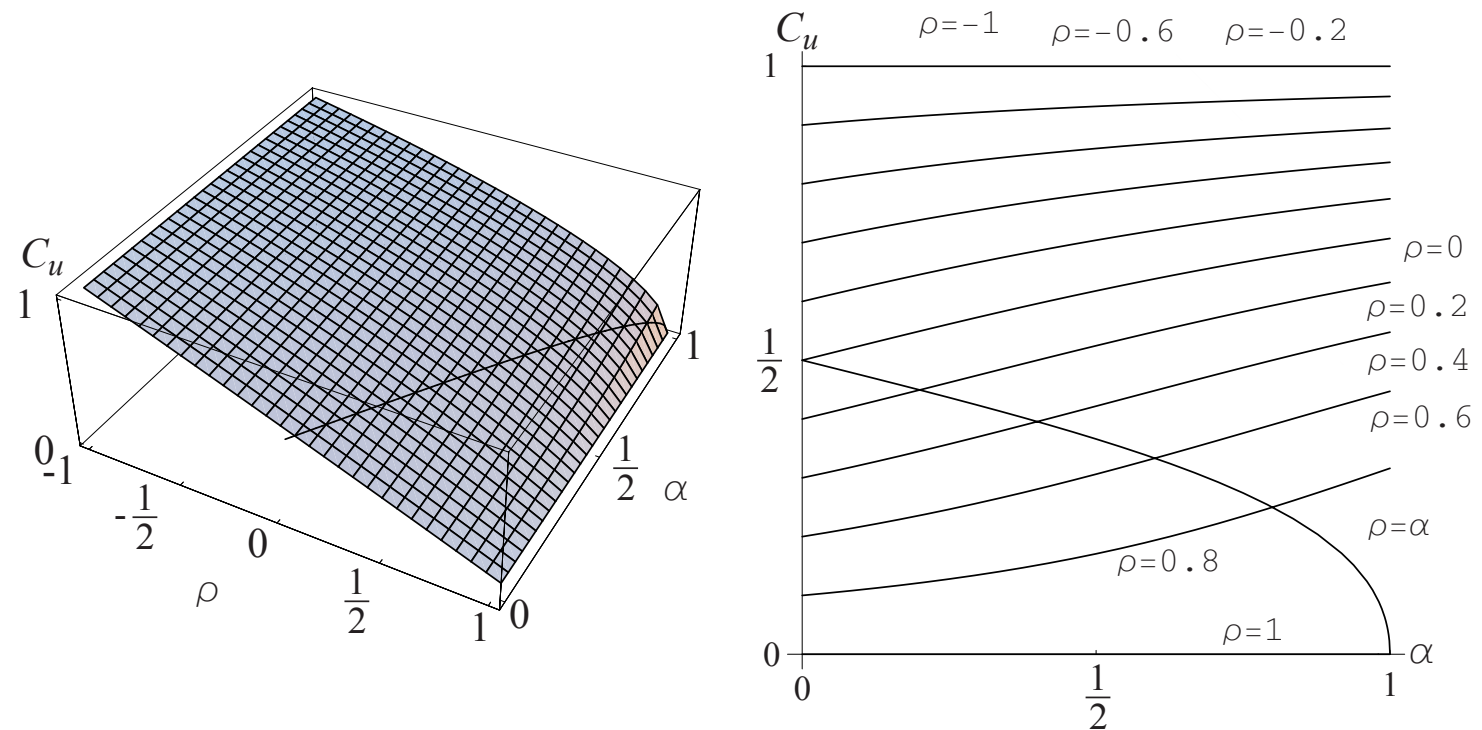

Figure 6. Spatial correlation, jobs-housing balance, and capacity utilization. 


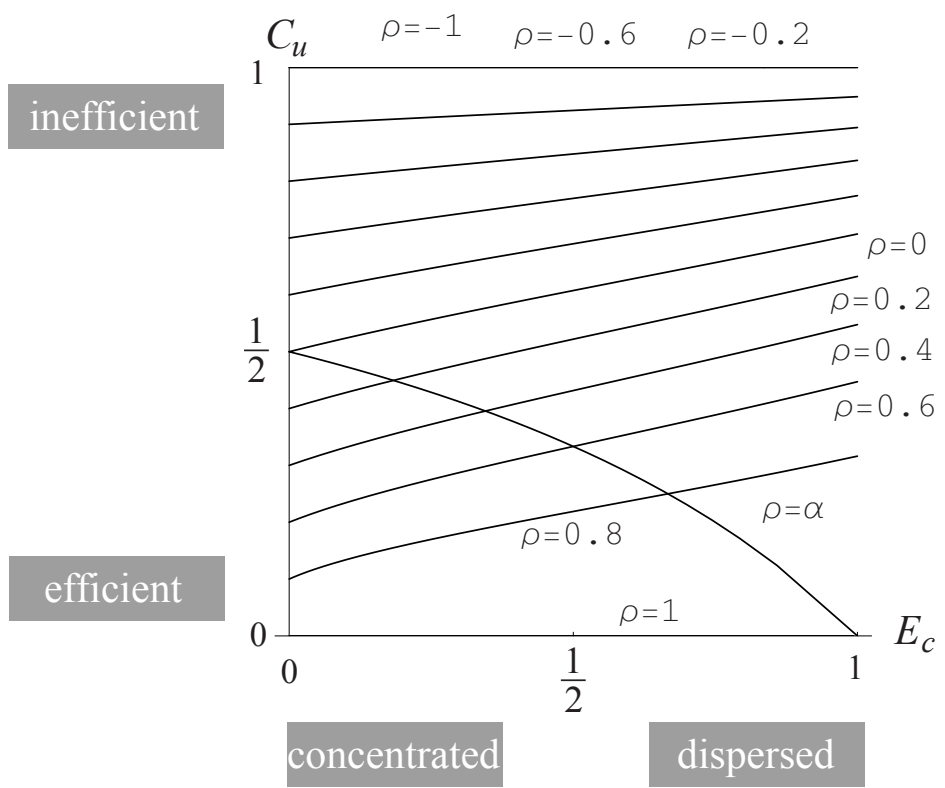

Figure 7. Relationship between excess commuting and capacity utilization. 


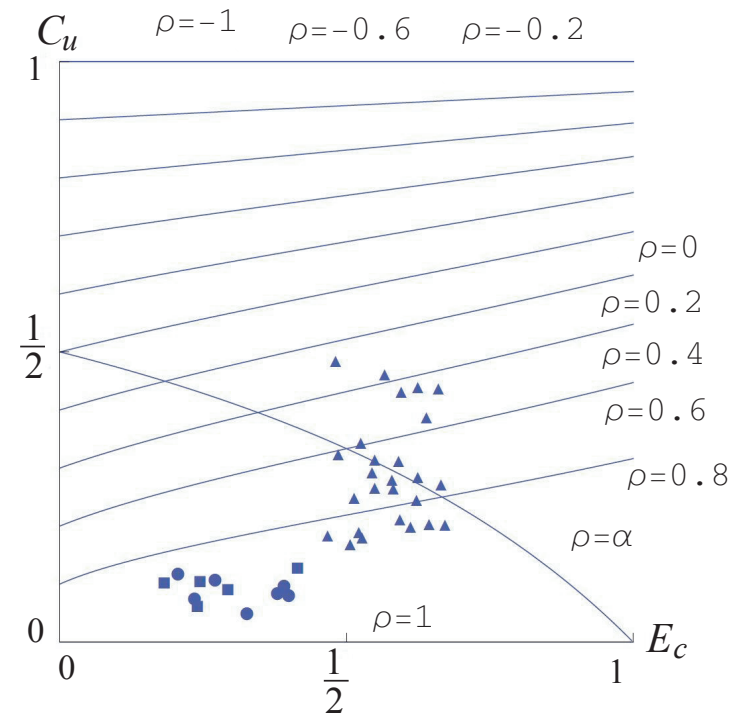

Figure 8. Excess and capacity utilization of US ( $\boldsymbol{\Delta})$, Japanese $(\boldsymbol{O})$, and Korean ( $\boldsymbol{\square})$ cities. 